\title{
Fields of values of linear pencils and spectral inclusion regions
}

\author{
Natália Bebiano, João da Providência, Ana Nata and João P. da Providência
}

\begin{abstract}
We propose efficient methods for the numerical approximation of the field of values of the linear pencil $A-\lambda B$, when one of the matrix coefficients $A$ or $B$ is Hermitian and $\lambda \in \mathbb{C}$. Our approach builds on the fact that the field of values can be reduced under compressions to the bidimensional case, for which these sets can be exactly determined. The presented algorithms hold for matrices both of small and large size. Furthermore, we investigate spectral inclusion regions for the pencil based on certain fields of values. The results are illustrated by numerical examples. We point out that the given procedures complement the known ones in the literature.
\end{abstract}

\section{Introduction}

Consider the linear pencil $A-\lambda B$, where $A$ and $B$ are $n \times n$ complex matrices and $\lambda \in \mathbb{C}$. The study of linear pencils has a rich and long history that goes back to Weierstrass and Kronecker in the nineteenth century, usually in the context of their spectral analysis. A complex number $\lambda$ is said to be an eigenvalue of the pencil if there exists a nonzero $x \in \mathbb{C}^{n}$ such that

Natália Bebiano

CMUC, University of Coimbra, Department of Mathematics, P 3001-454 Coimbra, Portugal

e-mail: bebiano@mat.uc.pt

João da Providência

CFisUC, University of Coimbra, Department of Physics, P 3004-516 Coimbra, Portugal

e-mail: providencia@teor.fis.uc.pt

Ana Nata

CMUC, Polytechnic Institute of Tomar, Department of Mathematics, P 2300-313 Tomar, Portugal e-mail: anata@ipt.pt

João P. da Providência

University of Beira Interior, Department of Physics, P 6201-001 Covilhã, Portugal

e-mail: joaodaprovidencia@daad-alumni.de 


$$
A x=\lambda B x .
$$

The vector $x$ is called an eigenvector of the pencil corresponding to the eigenvalue $\lambda$. The set of all eigenvalues is known as the spectrum of $A-\lambda B$ and denoted by $\sigma(A, B)$.

In the present work we are particularly interested in the numerical computation of certain fields of values, that are spectral inclusion regions for linear pencils. Motivations to investigate this problem come from stability theory and from the study of certain over-damped vibration systems, e.g. see [7].

The field of values of a linear pencil is denoted and defined as

$$
W(A, B)=\left\{\lambda \in \mathbb{C}: x^{*}(A-\lambda B) x=0, x \in \mathbb{C}^{n},\|x\|=1\right\},
$$

where $\|x\|=\langle x, x\rangle^{1 / 2}=\left(x^{*} x\right)^{1 / 2}$ is the usual Euclidean norm in $\mathbb{C}^{n}$ (cf. [10, 14, 12]). The set (2) does not contain the point at infinity. If $B$ is singular, then $\sigma(A, B)$ may have an infinite eigenvalue. Therefore, from the above definition, $W(A, B)$ is not necessarily a spectral inclusion region for the generalized eigenvalue problem (1). So, we consider a slightly modified definition: if $A, B$ have a common null space, then $W(A, B)=\mathbb{C} \cup\{\infty\}$; otherwise

$$
W(A, B)=\left\{\frac{x^{*} A x}{x^{*} B x}: x \neq 0\right\},
$$

where $1 / 0$ is understood as the point at infinity. When $B=I$, (3) reduces to the classical field of values of the $n \times n$ matrix $A$,

$$
W(A)=\left\{x^{*} A x:\|x\|=1\right\},
$$

that has been extensively investigated; see, for instance, [8].

Psarrakos [14] investigated the problem of the numerical computation of $W(A, B)$, when one of the coefficients $A$ or $B$ is Hermitian. His approach uses the algorithm of Li and Rodman [11] to compute boundary points $(u, v, w)$ of the so-called joint numerical range

$$
J N R(B, H, S)=\left\{\left(x^{*} B x, x^{*} H x, x^{*} S x\right): x \in \mathbb{C}^{n}, \text { with } x^{*} x=1\right\},
$$

where $A=H+i S$ and $H$ and $S$ are Hermitian. Given a point $(u, v, w)$ of $J N R(B, H, S)$ the solutions of the equations $u \lambda+v+i w=0,(u \neq 0)$ are points of $W(A, B)$. Psarrakos method performs specially well for matrices of small size. So, for large matrices, there is place for improvement and this is one of our main concerns. Our second goal is to obtain eigenvalue inclusion regions for linear matrix pencils, based on fields of values.

If $B$ is Hermitian positive definite (HPD), we clearly have $W(A, B)=$ $W\left(B^{-1 / 2} A B^{-1 / 2}\right)$ and due to the convexity of the classical field of values (stated by the Toeplitz-Hausdorff Theorem [6]), $W(A, B)$ is a convex set. However, $W(A, B)$ is not always convex and not even bounded or connected [10]. If $0 \in W(B)$, then 
$W(A, B)$ is unbounded and consequently this set is not an informative spectral inclusion region for the pencil. This motivated the investigation of other inclusion regions of field of values type. If $B$ is nonsingular, the spectrum of $B^{-1} A$ coincides with that of the pencil $A-\lambda B$. Henceforth, $W\left(B^{-1} A\right)$ and $W\left(A B^{-1}\right)$ are inclusion regions for the eigenvalues of (1). Interchanging the roles of $A$ and $B$ and considering the generalized eigenvalue problem $B x=\lambda^{-1} A x$, the sets $1 / W\left(A^{-1} B\right)$ and $1 / W\left(B A^{-1}\right)$, for nonsingular $A$, are also inclusion regions for (1). Division is interpreted elementwise.

The paper is organized as follows. In Sect. 2 we characterize the field of values of selfadjoint linear pencils, i.e., with Hermitian matrices as coefficients. In Sect. 3, auxiliary background is presented. In Sect. 4 we give a method to approximate $W(A, B)$ for Hermitian positive semi-definite $B$. In Sect. 5, a procedure to numerically approximate $W(A, B)$ for indefinite invertible $B$ is presented, based on the connection of this set with the Krein space field of values. Finally, in Sect. 6, some some conclusions are included. A few illustrative examples are provided. All images were computed numerically using MATLAB.

The key idea behind the algorithms here proposed is the following: we use subspace projection methods, a line of attack exploited by Hochstenbach in [7], stressing the fact that the field of values is often well approximated from a low dimension Krylov space. Our attempts are in this vein, and in summary, their advantages over the existing ones are that we perform projections on bidimensional spaces, in which case the fields of values are easily and exactly determined.

\section{Linear pencils with Hermitian coefficients}

In the sequel, $M_{n}$ denotes the algebra of $n \times n$ complex matrices. Throughout, we assume that the matrices $A$ and $B$ have no common nonzero isotropic vectors, i.e., $x^{*} A x=0$ and $x^{*} B x=0$, and so $W(A, B) \neq \mathbb{C}$.

The shape of $W(A, B)$ when $A$ and $B$ are Hermitian is described in Theorem 4.1 of [10]. Since the statement of this theorem is not correct, and is incorrectly reproduced in [14, Theorem 9], we include the proper result and proof.

Theorem 1. Let $A-\lambda B$ be a $n \times n$ self-adjoint pencil with $W(A, B) \neq \mathbb{C}$.

a) If $B$ is positive or negative definite, then $W(A, B)$ is a closed interval in $\mathbb{R}$.

b) If $B$ is positive (or negative) semi-definite, then 2 possibilities occur for $W(A, B)$ :

1) an unbounded interval of the form $[a,+\infty[$ or $]-\infty, a]$.

2) $\mathbb{R}$.

c) If $B$ is indefinite and $A$ is positive (negative) definite, then $W(A, B)$ is the union of 2 disjoint unbounded intervals and $0 \notin W(A, B)$.

d) If $B$ is indefinite and $A$ is semi-definite positive (or negative), then one of the following holds

1) $W(A, B)=\mathbb{R}$, 
2) $W(A, B)=]-\infty, a] \cup[0,+\infty[$ with $a<0$,

3) $W(A, B)=]-\infty, 0] \cup[b,+\infty[$ with $0<b$.

e) If both $B$ and $A$ are indefinite, then two possibilities may occur:

1) $W(A, B)=]-\infty, a] \cup[b,+\infty[$, with $0 \in W(A, B)$.

2) $W(A, B)=\mathbb{R}$.

In all cases, the endpoints of the intervals are eigenvalues of the pencil.

Proof. a) Observing that $W(A, B)=W\left(B^{-1 / 2} A B^{-1 / 2}\right)$, the result follows, because the classical field of values of a Hermitian matrix is a line segment whose endpoints are eigenvalues of the matrix (cf. [6]).

b) There exists a nonsingular matrix $T=U \oplus V, U \in M_{r}, V \in M_{n-r}$, such that

$$
T^{*} B T=I_{r} \oplus 0_{n-r}, \quad T^{*} A T=\left[\begin{array}{ll}
A_{11} & A_{12} \\
A_{21} & A_{22}
\end{array}\right]
$$

where

$$
A_{11}=\operatorname{diag}\left(\alpha_{1}, \ldots, \alpha_{r}\right), A_{22}=\operatorname{diag}\left(\alpha_{r+1}, \ldots, \alpha_{n}\right) .
$$

Since $W\left(\left(T^{*} A T, T^{*} B T\right)\right)=W(A, B)$, without loss of generality we may assume that $T=I_{n}$.

Now, firstly, we prove 1). Let us assume that $A_{22}$ is positive (negative) definite. Denote by $e_{j}$ the vector obtained from the null vector by replacing the $i$ th zero by 1 . Let $\beta$ be the smallest (largest) eigenvalue of the pencil $A-\lambda B$, and $u$ an associated eigenvector. Notice that, for $v=u+\gamma e_{r+1}, \gamma \in \mathbb{C}$, we have

$$
\frac{v^{*} A v}{v^{*} B v}=\beta+|\gamma|^{2} \frac{\alpha_{r+1}}{v^{*} B v} \text {. }
$$

Then,

$$
\left\{\frac{v^{*} A v}{v^{*} B v}: v=u+\gamma e_{r+1}, \gamma \in \mathbb{C}, v^{*} B v \neq 0\right\}=[\beta,+\infty[,
$$

and 1) follows. If $A_{22}$ and $B$ are both negative definite, the same conclusion holds. Otherwise, $W(A, B)=]-\infty, \beta]$.

Consider now that $A_{22}$ is indefinite, either nonsingular or singular. If $A_{22}$ is indefinite nonsingular, assume that $\alpha_{r+1} \alpha_{r+2}<0$ and take the compressions of the pencil to the subspaces spanned by $e_{1}, e_{r+1}$ and by $e_{1}, e_{r+2}$, respectively:

$$
(A-\lambda B)_{e_{1} e_{r+1}}=\left[\begin{array}{cc}
\alpha_{1}-\lambda & a_{1, r+1} \\
a_{r+1,1} & \alpha_{r+1}
\end{array}\right], \quad(A-\lambda B)_{e_{1} e_{r+2}}=\left[\begin{array}{cc}
\alpha_{1}-\lambda & a_{1, r+2} \\
a_{r+2,1} & \alpha_{r+2}
\end{array}\right]
$$

It is clear that

$$
W\left(A_{e_{1} e_{r+1}}, B_{e_{1} e_{r+1}}\right) \cup W\left(A_{e_{1} e_{r+2}}, B_{e_{1} e_{r+2}}\right)=\mathbb{R} \text {. }
$$

If $A_{22}$ is singular, assume that $\alpha_{r+1}=0$. Since the matrices $A$ and $B$ have no common nonzero isotropic vectors, then $a_{j, r+1} \neq 0,1 \leq j \leq r$. Assume that $a_{r+1,1} \neq 0$ and 
take the compression of the pencil to the subspace spanned by $e_{1}, e_{r+1}$,

$$
(A-\lambda B)_{e_{1} e_{r+1}}=\left[\begin{array}{cc}
\alpha_{1}-\lambda & a_{1, r+1} \\
a_{r+1,1} & 0
\end{array}\right] .
$$

It is easy to verify that

$$
W\left((A, B)_{e_{1} e_{r+1}}\right)=\mathbb{R}
$$

which proves b)2).

Similarly, we prove c), d), e).

We notice that the half ray in b)1) is of the form $[a,+\infty)$ if $B$ and $A_{22}$ in (5) are both positive definite or negative definite. Otherwise, the half ray is of the form $(-\infty, a]$.

\section{Background}

The classical field of values may be characterized as a union of elliptical disks. This result is many times referred as the Marcus-Pesce Theorem [13], although it was already known long before. In the following, we recall the standard compression of $W(A, B)$ into the fields of values of $2 \times 2$ pencils [4].

Theorem 2. (Chien and Nakazato) For any $A, B \in M_{n}$,

$$
W(A, B)=\bigcup_{u, v} W\left(A_{u v}, B_{u v}\right)
$$

where $u$ and $v$ vary over all pairs of orthonormal vectors in $\mathbb{C}^{n}$ and

$$
A_{u v}=\left[\begin{array}{cc}
\langle A u, u\rangle & \langle A v, u\rangle \\
\langle A u, v\rangle & \langle A v, v\rangle
\end{array}\right], \quad B_{u v}=\left[\begin{array}{cc}
\langle B u, u\rangle & \langle B v, u\rangle \\
\langle B u, v\rangle & \langle B v, v\rangle
\end{array}\right] .
$$

When $B$ is Hermitian positive definite, then also $B_{u v}$ is Hermitian positive definite, because it is a principal submatrix of a positive definite matrix. The field of values $W(A, B)$ in the 2 by 2 case, can be easily drawn from the entries of the matrices according to (for a proof, see e.g. [8]):

Theorem 3. (Elliptical Range Theorem) Let $A, B \in M_{2}$, with Hermitian positive definite $B$. Then $W(A, B)$ is a (possibly degenerate) closed elliptical disc, whose foci are the eigenvalues of $B^{-1} A, \lambda_{1}$ and $\lambda_{2}$. The equation of the ellipse is

$$
\frac{X^{2}}{M^{2}}+\frac{Y^{2}}{N^{2}}=\frac{1}{4}
$$

where

$$
X=(x-\operatorname{Re} c) \cos \gamma-(y-\operatorname{Im} c) \sin \gamma, Y=(x-\operatorname{Re} c) \sin \gamma+(y-\operatorname{Im} c) \cos \gamma,
$$


$c=\left(\lambda_{1}+\lambda_{2}\right) / 2$ is the center of the ellipse, and $\gamma$ is the slope of the major axis. The length of the major axis is $M=\sqrt{\operatorname{Tr}\left(A^{*} B^{-1} A B^{-1}\right)-2 \operatorname{Re}\left(\bar{\lambda}_{1} \lambda_{2}\right)}$, and $N=\sqrt{\operatorname{Tr}\left(A^{*} B^{-1} A B^{-1}\right)-\left|\lambda_{1}\right|^{2}-\left|\lambda_{2}\right|^{2}}$. is the length of the minor axis.

If $B$ is indefinite Hermitian, then $B_{u v}$ in (6) may be definite or indefinite. The following result treats this case [1].

Theorem 4. (Hyperbolical Range Theorem) Let $A, B \in M_{2}$ with $B$ indefinite Hermitian. Then $W(A, B)$ is bounded by a hyperbola with foci at $\lambda_{1}$ and $\lambda_{2}$, the eigenvalues of $B^{-1} A$, and transverse and non-transverse axis of length

$M=\sqrt{\operatorname{Tr}\left(B^{-1} A^{*} B^{-1} A\right)-2 \operatorname{Re}\left(\lambda_{1} \bar{\lambda}_{2}\right)}$ and $N=\sqrt{\left|\lambda_{1}\right|^{2}+\left|\lambda_{2}\right|^{2}-\operatorname{Tr}\left(B^{-1} A^{*} B^{-1} A\right)}$.

Consider $W(A, B)$ for $A, B \in M_{2}$, with $B$ positive (negative) semidefinite. Observing that

$$
W\left(\mathrm{e}^{i \phi}(A+\zeta B), k B\right)=\frac{1}{k} \mathrm{e}^{i \phi}(W(A, B)+\zeta), \quad k, \phi \in \mathbb{R}, \zeta \in \mathbb{C},
$$

without loss of generality, we can take

$$
B=\operatorname{diag}(1,0), \quad A=\left[\begin{array}{cc}
a \mathrm{e}^{i \gamma} & c \mathrm{e}^{i \gamma} \\
d & b
\end{array}\right], \quad c, d \geq 0, b>0, a=\frac{c d}{b} .
$$

Notice that $W(A, B)=\mathbb{C}$ if $b=0$.

Theorem 5. (Parabolical Range Theorem) Let $A, B \in M_{2}$ be of the form (7). Then $W(A, B)$ is bounded by the (possibly degenerate) parabola with focus $\lambda_{0}=0$ and equation

$$
\frac{y^{2}}{4 p^{2}}-\frac{x}{p}=1
$$

where

$$
p=\frac{a^{2} b^{2}+c^{4}-2 a b c^{2} \cos \gamma}{4 b c^{2}} .
$$

Proof. By Theorem 1.4 i) and ii) of [4], $W(A, B)$ is unbounded convex. Let a supporting line of $W(A, B)$ be given by $x \cos \theta+y \sin \theta+w=0$. Writing $A=H(A)+i K(A)$, with $H(A)=\left(A+A^{*}\right) / 2$ and $K(A)=\left(A-A^{*}\right) /(2 i)$, Kippenhan's Theorem [9] asserts that $\operatorname{det}(\cos \theta H(A)+\sin \theta K(A)+w B)=0$. We find

$$
\operatorname{det}(\cos \theta H(A)+\sin \theta K(A)+w B)=\frac{1}{4}\left(-\frac{a^{2} b^{2}}{c^{2}}-c^{2}+2 a b \cos \gamma+4 b w \cos \theta\right),
$$

with $\theta \in[0,2 \pi[$. Replacing $w$ by the root of the above characteristic polynomial, we get

$$
x \cos \theta+y \sin \theta+\frac{1}{4}\left(\frac{a^{2} b}{c^{2}}+\frac{c^{2}}{b}-2 a \cos \gamma\right) \sec \theta=0 .
$$

The parametric equations of the envelope of this family of lines are given by 


$$
\begin{gathered}
x=\frac{2 a b c^{2} \cos \gamma-a^{2} b^{2}-c^{4}}{4 b c^{2}} \cos (2 \theta) \sec ^{2} \theta \\
y=\frac{2 a b c^{2} \cos \gamma-a^{2} b^{2}-c^{4}}{2 b c^{2}} \tan \theta,
\end{gathered}
$$

from which the desired result follows.

\subsection{Connection of $W(A, B)$ with the Krein space field of values for indefinite Hermitian $B$}

There is an interesting relation of $W(A, B)$ when $B$ is indefinite Hermitian, with the Krein space field of values [2,3]. Indeed, suppose that $B$ is an $n \times n$ indefinite Hermitian matrix with inertia $(r, n-r)$. Consider $\mathbb{C}^{n}$ endowed with indefinite inner product $[x, y]=y^{*} B x, x, y \in \mathbb{C}^{n}$. The Krein space field of values of $A \in M_{n}$ is defined by

$$
W_{B}(A)=\left\{\frac{[A w, w]}{[w, w]}: w \in \mathbb{C}^{n},[w, w] \neq 0\right\} .
$$

We easily find the connection of $W_{B}(A)$ with the field of values of the pencil $B A-\lambda B$. Indeed, we easily get

$$
W_{B}(A)=W(B A, B)=\left\{\frac{\langle B A w, w\rangle}{\langle B w, w\rangle}: w \in \mathbb{C}^{n},\langle B w, w\rangle \neq 0\right\},
$$

and so, $W(A, B)=W_{B}\left(B^{-1} A\right)$.

\section{Approximation of $W(A, B)$ for positive semidefinite $B$}

\subsection{Algorithm 1. Approximation of $W(A, B), B$ HPSD}

Input: A matrix $A \in M_{n}$, a Hermitian positive semidefinite matrix $B$ and $m$ angles. Output: An approximation for $W(A, B)$.

1. Set $\theta_{k}=(k-1) \pi / m, k=1, \ldots, m+1$ for some positive integer $m \geq 3$.

2. Starting with $k=1$ and up to $k=m$, take the following steps:

(i) Compute an eigenvector $u_{k}$ associated to $\lambda_{\min }\left(\Re\left(\mathrm{e}^{-i \theta_{k}} A\right)-\lambda B\right)$ if $W\left(\Re\left(\mathrm{e}^{-i \theta_{k}} A\right), B\right)=\left[a,+\infty\left[\left(\right.\right.\right.$ to $\lambda_{\max }\left(\Re\left(\mathrm{e}^{-i \theta_{k}} A\right)-\lambda B\right)$ if $W\left(\Re\left(\mathrm{e}^{-i \theta_{k}} A\right), B\right)$ $=]-\infty, a])$.

(ii) Compute the compressions of $A$ and $B$ to $\operatorname{span}\left\{u_{k}, u_{k+1}\right\}$, denoted by $A_{\tilde{u}_{k} \tilde{u}_{k+1}}$ and $B_{\tilde{u}_{k}} \tilde{u}_{k+1}$.

(iii) Compute and draw the boundary of $W\left(A_{\tilde{u}_{k} \tilde{u}_{k+1}}, B_{\tilde{u}_{k}} \tilde{u}_{k+1}\right)$ denoted by $\Gamma_{k}$. 
(iv) If $k<m$, take next $k$ value and return to (i). Otherwise, continue.

3. Take the convex-hull of the collection of curves $\Gamma_{1}, \ldots, \Gamma_{m}$, as an approximation for $W(A, B)$.

Some observations are in order. According to the Elliptical and the Parabolical Range Theorems, the collection of curves in Step 3 of Algorithm 1 is constituted by ellipses and parabolas. We observe that, if the smallest and the largest eigenvalues of the pencil $\mathfrak{R}\left(\mathrm{e}^{-i \theta_{k}} A\right)-\lambda B$ are both infinite, then $W\left(\Re\left(\mathrm{e}^{-i \theta_{k}} A\right), B\right)=\mathbb{R}$ (cf. Theorem 1). Algorithm 1 may be applied to $B$ HPD with the following replacements of Sub-steps (i), (ii), (iii) of Step 2:

(i) Compute eigenvectors $u_{k}$ (or $\left.v_{k}\right)$ associated to $\lambda_{\min }\left(\Re\left(\mathrm{e}^{-i \theta_{k}} A\right)-\lambda B\right)$ $\left(\lambda_{\max }\left(\Re\left(\mathrm{e}^{-i \theta_{k}} A\right)-\lambda B\right)\right)$

(ii) Compute the compressions of $A$ to $\operatorname{span}\left\{u_{k}, u_{k+1}\right\}$ and $\operatorname{span}\left\{v_{k}, v_{k-1}\right\}$, denoted $A_{\tilde{u}_{k} \tilde{u}_{k+1}}$ and $A_{\tilde{v}_{k} \tilde{v}_{k-1}}$, and do the sane for $B$, notation: $B_{\tilde{u}_{k}} \tilde{u}_{k+1}$ and $B_{\tilde{v}_{k} \tilde{v}_{k-1}}$.

(iii) Compute and draw the boundary of $W\left(A_{\tilde{u}_{k} \tilde{u}_{k+1}}, B_{\tilde{u}_{k}} \tilde{u}_{k+1}\right)$ denoted by $\Gamma_{k}$ and the boundary of $W\left(A_{\tilde{v}_{k} \tilde{v}_{k-1}}, B_{\tilde{v}_{k} \tilde{v}_{k-1}}\right)$ denoted by $\Lambda_{k}$.

and the following replacement of Step 3:

3. Take the convex-hull of the collection of curves $\Gamma_{1}, \ldots, \Gamma_{m}, \Lambda_{1}, \ldots, \Lambda_{m}$ as an approximation for $W(A, B)$.

Example 1. We take the matrix $A=C_{1} \oplus i C_{2}$ and the positive definite matrix $B=I_{20}+0.1 D D^{*}$, with $C_{1}=\operatorname{randn}(10), C_{2}=\operatorname{randn}(10), D=\operatorname{randn}(20)$ with $m=6$. See Fig. 1. The Zoom shows that the bounded complements of $1 / W\left(A^{-1} B\right)$ and $1 / W\left(B A^{-1}\right)$ are spectral exclusion regions for the eigenvalues of the pencil.

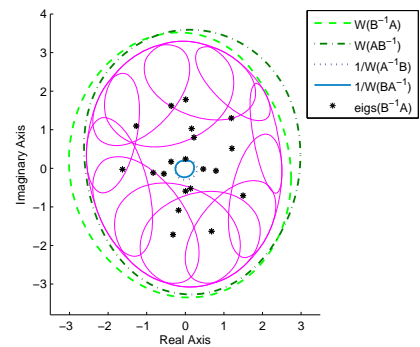

(a) $A=C_{1} \oplus i C_{2}, B=I_{20}+0.1 D D^{*}$.

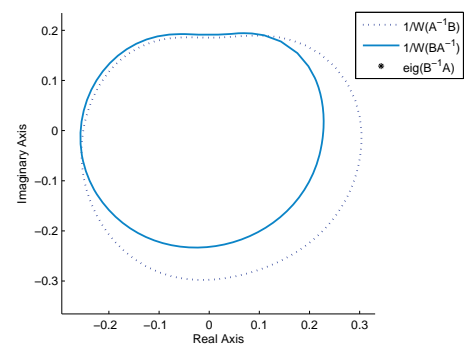

(b) Zoom-in of (a)

Fig. 1 (a) Eigenvalues of $A-\lambda B$ (asterisks) and the boundaries of $W(A, B), W\left(B^{-1} A\right), W\left(A B^{-1}\right)$, $1 / W\left(A B^{-1}\right), 1 / W\left(B^{-1} A\right)$, for Example 1, $m=6$. (b) Exclusion regions for the eigenvalues of the pencil, $1 / W\left(A B^{-1}\right), 1 / W\left(B^{-1} A\right)$.

Example 2. We take the matrix $A=\operatorname{randn}(20)$, and the positive semidefinite matrix $B=I_{19} \oplus 0_{1}$. We carry out Algorithm 1 with $m=6$. Considered as a spectral inclusion region, $W(A, B)$ has drawbacks since it is unbounded. See Fig. 2. 


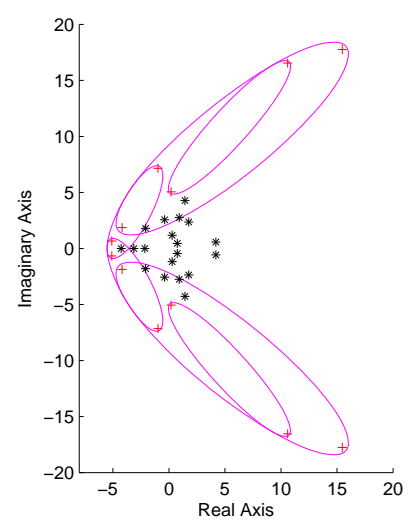

Fig. 2 Eigenvalues of $A-\lambda B$ (asterisks) and part of the boundary of $W(A, B)$. Here, $B$ is PSD. (Example 2).

\section{Approximation of $W(A, B)$ for indefinite Hermitian $B$}

Recall that we are assuming that the matrices $A$ and $B$ have no common nonzero isotropic vectors, and $x^{*} B x=0$, and so $W(A, B) \neq \mathbb{C}$. We may define

$$
\begin{aligned}
& W_{+}(A, B)=\left\{\lambda \in \mathbb{C}: u^{*} A u-\lambda u^{*} B u=0, u \in \mathbb{C}^{n}, u^{*} B u>0\right\}, \\
& W_{-}(A, B)=\left\{\lambda \in \mathbb{C}: u^{*} A u-\lambda u^{*} B u=0, u \in \mathbb{C}^{n}, u^{*} B u<0\right\},
\end{aligned}
$$

and so $W(A, B)=W_{+}(A, B) \cup W_{-}(A, B)$. We also define, if $A$ is Hermitian,

$$
\begin{aligned}
& \sigma^{+}(A, B)=\left\{\lambda \in \mathbb{C}: A u-\lambda B u=0, u \in \mathbb{C}^{n}, u^{*} B u>0\right\} \\
& \sigma^{-}(A, B)=\left\{\lambda \in \mathbb{C}: A u-\lambda B u=0, u \in \mathbb{C}^{n}, u^{*} B u<0\right\} .
\end{aligned}
$$

To avoid trivial cases of degeneracy of $W(A, B)$, we shall be specially concerned with the class of matrices in $M_{n}$, for which there exists a real interval $\left[\theta_{1}, \theta_{2}\right]$, with $0<\theta_{2}-\theta_{1}<\pi$, such that for $\theta$ ranging over that interval, the Hermitian pencil

$$
H\left(\mathrm{e}^{-i \theta} A\right)-\lambda B
$$

has real eigenvalues satisfying simultaneously the following conditions:

(i) $\lambda_{1}\left(H\left(\mathrm{e}^{-i \theta} A\right)-\lambda B\right) \geq \cdots \geq \lambda_{r}\left(H\left(\mathrm{e}^{-i \theta} A\right)-\lambda B\right) \in \sigma^{+}\left(H\left(\mathrm{e}^{-i \theta} A\right), B\right)$;

(ii) $\lambda_{r+1}\left(H\left(\mathrm{e}^{-i \theta} A\right)-\lambda B\right) \geq \cdots \geq \lambda_{n}\left(H\left(\mathrm{e}^{-i \theta} A\right)-\lambda B\right) \in \sigma^{-}\left(H\left(\mathrm{e}^{-i \theta} A\right), B\right)$;

(iii) $\lambda_{r}\left(H\left(\mathrm{e}^{-i \theta} A\right)-\lambda B\right)>\lambda_{r+1}\left(H\left(\mathrm{e}^{-i \theta} A\right)-\lambda B\right)$. 
For the pencils of this class, $W\left(H\left(\mathrm{e}^{-i \theta} A\right), B\right)$ is non-degenerate, that is, it is not a singleton, a whole line (possibly without a point), or the whole complex plane (possibly without a line). This class of pencils is called class $\mathscr{N} \mathscr{D}$, the acronym for non-degenerate.

When $B$ is indefinite Hermitian nonsingular, $B_{u v}$ may be indefinite or definite. If $B_{u v}$ is indefinite, Theorem 4 holds and $\partial W\left(A_{u v}, B_{u v}\right)$, the boundary of $W\left(A_{u v}, B_{u v}\right)$, is the union of two hyperbolical arcs, one in $W_{+}\left(A_{u v}, B_{u v}\right)$ and the other in $W_{-}\left(A_{u v}, B_{u v}\right)$. If $B_{u v}$ is definite, $\partial W\left(A_{u v}, B_{u v}\right)$ may be in $W_{+}(A, B)$ or in $W_{-}(A, B)$. Let the curves $C_{1}^{+}, C_{2}^{+}, \ldots, C_{r}^{+}\left(C_{1}^{-}, C_{2}^{-}, \ldots, C_{s}^{-}\right)$denote the arcs of $\partial W\left(A_{u v}, B_{u v}\right)$ in $W_{+}(A, B)\left(W_{-}(A, B)\right)$. Let $K^{+}=\operatorname{conv}\left(C_{1}^{+}, C_{2}^{+}, \ldots, C_{r}^{+}\right), K^{-}=$ conv $\left(C_{1}^{-}, C_{2}^{-}, \ldots, C_{s}^{-}\right)$. The pseudo-convex hull of $C_{1}^{+}, C_{2}^{+}, \ldots, C_{r}^{+}, C_{1}^{-}, C_{2}^{-}, \ldots, C_{s}^{-}$, denoted pconv $\left(C_{1}^{+}, C_{2}^{+}, \ldots, C_{r}^{+}, C_{1}^{-}, C_{2}^{-}, \ldots, C_{s}^{-}\right)$, is the union of all half-rays of the lines passing through $z^{+} \in K^{+}, z^{-} \in K^{-}$with endpoint in $z^{+}$not containing $z^{-}$, or with endpoint in $z^{-}$not containing $z^{+}$.

As a preliminary stage to Algorithm 2, we start by searching an admissible angle $\theta$. If the matrix is complex, we test the angle $-\pi / 2$ for this property. If the answer is positive, we go to Step 0 . If not, we test the admissibility of $\theta=0$. In the affirmative case, we proceed to Step 0 . Otherwise, test the admissibility of the angles

$$
\theta_{\ell, k}=-2^{k-1} \pi / 2^{k}+(2 \ell-1) \pi / 2^{k}, \ell=0,1, \ldots, 2^{k-1},
$$

until an admissible angle is found, and then we proceed to Step 0. It is worth noticing that replacing the matrix $A$ by $\mathrm{e}^{-i \theta_{\ell, k}} A$, where $\theta_{\ell, k}$ is admissible for the pencil $H(A)-\lambda B$, then $\theta=0$ is admissible for the rotated pencil

$$
H\left(\mathrm{e}^{-i \theta_{\ell, k}} A\right)-\lambda B
$$

Step 0. Choice of $\left[\theta_{\min }, \theta_{\max }\right]$ Fix a tolerance $t o l=\pi / 2^{N}, N \geq 4$ and let $\theta=0$ be an admissible angle. Starting with $\theta_{0}=0$, construct a set of admissible angles, as follows. Bisect successively the interval $[0, \pi / 2]$ until we find an admissible angle $\theta_{1}=\pi / 2^{v_{1}}$, the integer $v_{1}$ being such that the angle $\theta_{1}+\pi / 2^{v_{1}}$ is non-admissible. Proceed in this way until we find a new admissible angle $\theta_{2}=\pi / 2^{v_{1}}+\pi / 2^{v_{1}+v_{2}}$, the integer $v_{2}$ being such that the angle $\theta_{2}+\pi / 2^{v_{1}+v_{2}}$ is non-admissible, and so on, until we reach the admissible angle $\theta_{k}=\pi / 2^{v_{1}}+\pi / 2^{v_{1}+v_{2}}+\cdots+\pi / 2^{v_{1}+v_{2}+\ldots+v_{k}}$, such that $\theta_{k}+\pi / 2^{v_{1}+v_{2}+\cdots+v_{k}}$ is non-admissible, being $v_{1}+v_{2}+\cdots+v_{k} \leq N$. The admissible angles $\bar{\theta}_{1}=-\pi / 2^{\bar{v}_{1}}, \bar{\theta}_{2}=-\pi / 2^{\bar{v}_{1}}-\pi / 2^{\bar{v}_{1}+\bar{v}_{2}}, \ldots, \bar{\theta}_{\ell}=-\pi / 2^{\bar{v}_{1}}-$ $\pi / 2^{\bar{v}_{1}+\bar{v}_{2}}-\cdots-\pi / 2^{\bar{v}_{1}+\bar{v}_{2}+\cdots+\bar{v}_{\ell}}$ are analogously obtained. If the matrix is real, we obviously have $\bar{\theta}_{j}=-\theta_{j}, j=1, \cdots k$. The interval of admissible angles is $\left[\theta_{\min }, \theta_{\max }\right]=\left[\bar{\theta}_{\ell}, \theta_{k}\right]$. 


\subsection{Algorithm 2. Approximation of $W(A, B)$, for indefinite Hermitian $B$}

Input: A matrix $A \in M_{n}$, an indefinite Hermitian nonsingular matrix $B$ and $m$ angles. Output: An approximation for $W(A, B)$.

1. Set $\theta_{k}=\theta_{\min }+\frac{k-1}{m}\left(\theta_{\max }-\theta_{\min }\right), k=1, \ldots, m+1$ for some positive integer $m \geq 3$.

2. Starting with $k=1$ and up to $k=m$, take the following steps:

(i) Compute eigenvectors $u_{k}$ and $v_{k}$ associated, respectively, to

$$
\lambda_{\text {max }}\left(H\left(\mathrm{e}^{-i \theta} A\right)-\lambda B\right) \in \sigma_{-}\left(\Re\left(\mathrm{e}^{-i \theta_{k}} A\right), B\right)
$$

and

$$
\lambda_{\text {min }}\left(H\left(\mathrm{e}^{-i \theta} A\right)-\lambda B\right) \in \sigma_{+}\left(\Re\left(\mathrm{e}^{-i \theta_{k}} A\right), B\right) .
$$

(ii) Compute the compressions of $A$ and $B$ to $\operatorname{span}\left\{u_{k}, u_{k+1}\right\}$ and $\operatorname{span}\left\{v_{k}, v_{k+1}\right\}$, $A_{\tilde{u}_{k} \tilde{u}_{k+1}}, A_{\tilde{v}_{k} \tilde{v}_{k+1}}, B_{\tilde{u}_{k} \tilde{u}_{k+1}}$ and $B_{\tilde{v}_{k} \tilde{v}_{k+1}}$, respectively.

(iii) Compute and draw $\partial W\left(A_{\tilde{u}_{k} \tilde{u}_{k+1}}, B_{\tilde{u}_{k} \tilde{u}_{k+1}}\right)$ and $\partial W\left(A_{\tilde{v}_{k} \tilde{v}_{k+1}}, B_{\tilde{v}_{k} \tilde{v}_{k+1}}\right)$, denoted by $\Gamma_{k}$ and $\Lambda_{k}$, respectively.

(iv) If $k<m$, take next $k$ value and return to (i). Otherwise, continue.

3. Take the pseudo-convex-hull of the collection of curves $\Gamma_{1}, \ldots, \Gamma_{m}, \Lambda_{1}, \ldots, \Lambda_{m}$ as an approximation for $W(A, B)$.

We now present an illustrative example.

Example 3. The fields of values $W(A, B)$ and $W\left(B^{-1} A\right)$, where $A=\operatorname{randn}(20)+7 I_{20}$ and $B=I_{10} \oplus-I_{10}$, have been obtained using Algorithm 2 and are plotted in Fig. 3 . We have used $\theta_{\max }=-\theta_{\min }=0.5915413$ and $m=6$. To compare, in accuracy, Algorithm 2 with Psarrakos Algorithm, we have computed the area of the domain bounded by the obtained approximation of $\partial W(A, B)$ and by the lines parallel to the imaginary axis with abscissas $x=8$ and $x=-8$. We have also considered higher values of $m$ in order to improve the accuracy. As Table 1 shows, Algorithm 2 requires much fewer eigenanalyses and reaches faster a given number of accurate digits.

\section{Conclusions}

We have given procedures to numerically approximate $W(A, B)$, of which at least one of the two matrices is Hermitian. In our approach we used the key fact that the field of values of a linear pencil is efficiently approximated by the compression into bidimensional linear pencils. Our algorithms compute the extreme eigenvalues of a small number of rotated pencils $H\left(\mathrm{e}^{-i \theta_{j}} A\right)-\lambda B$ together with the respective eigenvectors $u_{j}$. In a second stage compression matrices of size 2 for the $\operatorname{span}\left\{u_{j}, u_{j+1}\right\}$ 


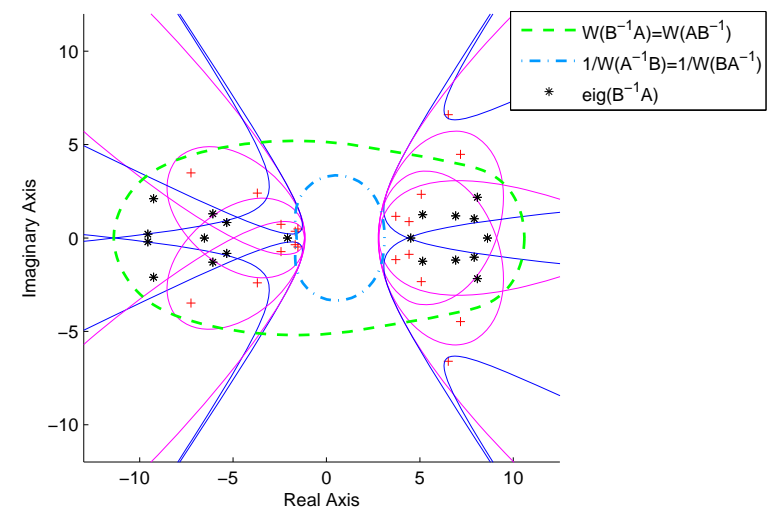

Fig. 3 Field of values $W(A, B)$, eigenvalues of the pencil (asterisks), boundaries of $W\left(B^{-1} A\right)$ (green) and of $1 / W\left(B^{-1} A\right)$ (blue) for $A=\operatorname{randn}(20)+7 I_{20}, B=I_{10} \oplus-I_{10} m=6$.

Table 1 Performance of Algorithm 2 and Psarrakos Algorithm [14], for the matrix of Example 3. The computed area is the one of the domain bounded by the approximation of $\partial W(A, B)$ and by the vertical lines $x=-8$ and $x=8$.

\begin{tabular}{|c|c|c|c|c|c|}
\hline & $m$ & eigenanalyses & area & acc. digits & seconds \\
\hline \hline Algorithm 2 & 6 & 24 & 160.7854 & 2 & 0.121777 \\
& 12 & 30 & 161.5071 & 3 & 0.230439 \\
& 24 & 42 & 161.6953 & 3 & 0.322045 \\
& 48 & 66 & 161.7327 & 5 & 0.494298 \\
& 96 & 114 & 161.7378 & 5 & 1.082117 \\
& 192 & 210 & 161.7391 & 6 & 2.783337 \\
\hline Psarrakos Algorithm & 6 & 602 & 159.2174 & 1 & 0.387542 \\
& 12 & 2354 & 160.6860 & 2 & 0.731580 \\
& 24 & 9314 & 161.5101 & 3 & 1.967806 \\
& 48 & 37058 & 161.6773 & 3 & 7.043684 \\
& 96 & 147842 & 161.7255 & 4 & 27.077536 \\
& 192 & 590594 & 161.7368 & 5 & 111.270553 \\
\hline
\end{tabular}

for each $j=2, \ldots, m$ are constructed. Elliptical and hyperbolical arcs generated from the compression matrices provide a quick and quite accurate approximation of the searched boundaries. Evaluating eigenvalues and eigenvectors involves $O\left(n^{3}\right)$ operations for $n$ sized matrices. Performing 2-by-2 compressions is an $O\left(n^{2}\right)$ process and determining ellipses, parabolas or hyperbolas by using Theorems 3, 4, 5 takes almost no time. Variations in relative speed and accuracy occur for varying dimensions, varying matrices and obviously changing the prescribed degree of accuracy. The preliminary stages for Algorithm 2 take negligible time. We stress that the proposed algorithms hold for both matrices of small and large dimensions. Psarrakos method [14] can be used for pairs of matrices of small dimension but it appears not to be interesting for large sized matrices. In fact, his method uses a discretization 
of the unit sphere in $\mathbb{R}^{3}$ and for each grid point a maximum eigenvalue of a certain associated Hermitian matrix has to be computed. Hochstenbach's Algorithm [7] applies only for Hermitian positive definite matrices $B$ (or any HPD linear combination of $A$ and $B$ ). We have also focused on spectral inclusion regions for matrix pencils based on fields of values.

It would be of interest to obtain accurate and fast algorithms to plot $W(A, B)$ whenever neither $A$ nor $B$ are Hermitian.

Acknowledgements The authors wish to thank the Referees for most valuable comments. This work was partially supported by the Centre for Mathematics of the University of CoimbraUID/MAT/00324/2013, funded by the Portuguese Government through FCT/MCTES and cofunded by the European Regional Development Fund through the Partnership Agreement PT2020.

\section{References}

1. Bebiano, N., Lemos, R., Providência, J. da, Soares, G.: On generalized numerical ranges of operators on an indefinite inner product space. Linear and Multilinear Algebra 52, 203-233 (2004)

2. Bebiano, N., Providência, J. da, Nata, A., Providência, J.P. da: Computing the numerical range of Krein space operators. Open Mathematics 13, 2391-5455 (2014)

3. Bebiano, N., Providência, J. da, Nata, A., Soares, G.: Krein spaces numerical ranges and their computer generation. Electronic Journal of Linear Algebra 17, 192-208 (2008)

4. Chien, M-T., Nakazato, H.: The numerical range of linear pencils of 2-by-2 matrices. Linear Algebra Appl. 341, 69-100 (2002)

5. Davis, C.: The Toeplitz-Hausdorff Theorem explained. Canad. Math. Bull. 14, 245-246 (1971)

6. Gustafson, K.E., Rao, D.K.M.: Numerical range, the field of values of linear operators and matrices. Springer-Verlag, New York (1997)

7. Hochstenbach, M.E.: Fields of values and inclusion regions for matrix pencils. Electronic Transactions on Numerical Analysis 38, 98-112 (2011)

8. Horn, R.A., Johnson, C.R.: Topics in matrix analysis. Cambridge University Press, Cambridge (1991)

9. Kippenhahn, R.: Über den wertevorrat einer matrix. Math. Nachr. 6, 193-228 (1951)

10. Li, C.-K., Rodman, L.: Numerical range of matrix polynomials. SIAM J. Matrix Anal. Appl. 15, 1256-1265 (1994)

11. Li, C.-K., Rodman, L.: Shapes and computer generation of numerical ranges of Krein space operators. Electron. J. Linear Algebra 3, 31-47 (1998)

12. Loghin, D., Guzen, M. van, Jonkers, E.: Bounds on the eigenvalue range and on the field of values of non-Hermitian and indefinite finite element matrices. J. Comput. Appl. Mat. 189 (1-2), 304-323 (2006)

13. Marcus, M., Pesce, C.: Computer generated numerical ranges and some resulting theorems. Linear and Multilinear Algebra. 20, 121-157 (1987)

14. Psarrakos, P.J.: Numerical range of linear pencils. Linear Algebra Appl. 317, 127-141 (2000)

15. The Matrix Market, a repository for test matrices. http://math.nist.gov/MatrixMarket 